\title{
Agenda des Réunions et Congrès
}

26/27 Février 1996

$2^{e ̀ m e}$ Congrès National de la Société Italienne d'Andrologie Médicale.

Florence, Italie

Contact : Dr G. ForTI - Unité d'Andrologie

Viale Pieracini 6 - 50139 Florence - Italie

Tél : 39-55-4377854; Fax : 39-55-4377290

\section{4/17 Mars 1996}

Marseille, Carrefour de la Sexologie :

- XXVIème Séminaire de Perfectionnement en Sexologie Clinique de l'Association Inter-Hospitalo-Universitaire de Sexologie

- IIIème Congrès de la Fédération Européenne de Sexologie

- IIèmes journées de la Société Européenne de Gynéco-Andro-Psychosomatique

Principaux thèmes : Cognitivisme et Sexologie - L'impuissance en Europe (les différences culturelles scientifiques et thérapeutiques vis à vis du diagnostic et de la prise en charge de l'impuissance dans les différents pays de la Communauté Européenne). Ethnosexologie - Médias et sexualité - Chirurgie Esthétique, image du corps et sexualité Ethique, sexualité et reproduction - L'inceste.

Contact : Secrétariat du Congrès

21, place Alexandre Labadié - 13001 Marseille

Tél : 91-95-76-96 ; Fax : 91-50-52-77

12/14 Avril 1996

1ère rencontre franco-marocaine : Société d'Andrologie de Langue Française

(SALF) - Société Marocaine d'Andrologie et de Sexologie (SMAS)

Palais des Congrès, Marrakech, Maroc

Principaux thèmes : Infertilité masculine - Ambiguités Sexuelles - Sexologie

Contact :

Maroc: Dr SmiRes-Bennani Azis

44, rue Abou Abdellah Nafii-Maârif

20100 Casablanca, Maroc

Tél : 25-95-75 ; Fax : 98-60-51

France : Dr M. SCHOUMAN

164, Av. Charles de Gaulle

92200 Neuilly

Tél : 47-47-02-02 ; Fax 47-47-47-34

14/19 Avril 1996

9th Européan Testis Workshop on Molecular and Cellular Endocrinology

Geilo, Norvège

Contact :

Pr V. Hansson - Institute for Medical Biochemistry

P.O. Box 1112, Blindern

$\mathrm{N}-0317$, Oslo, Norvège

25/28 Avril 1996

Tél : 47-22851002; Fax : 47-22851058

American Society of Andrology Annual Meeting

Minneapolis, Minnesota, USA

Contact : Dr Chris Gosh - ASA Executive Office

P.O. Box 15171, Lenexa

KS 66285-5171, USA

Tél : 913-541-9077 
VIth International Symposium on Andrology

Palma de Mallorca, Espagne

Contact : Dr M. Rossello Barbara - Centro de Urologia - Andrologia y Sexologia de Baleares - Calle Santiago Russinol - 9, 3aPlanta

07012 Palma de Mallorca, Espagne

Tél : 971-716600 ; Fax : 971-726519

8/10 Mai 1996

Journées de la Fédération Française d'Etude de la Reproduction

Palais des Congrès, Paris

Contact : Dr Joelle BELAISCH-ALLART - Service de Gynécologie-Obstétrique

Centre Hospitalier J. Rostand - 141 Grande Rue

92311 Sèvres Cedex

Tél : 42-26-94-35 ; Fax : 46-26-94-34

16/18 Mai 1996

First National Congress of Andrology with International Participation

Sofia, Bulgarie

Contact : Dr D. TzvetKou

13 Christo Kovachev

1504 Sofia, Bulgarie

Tél : 359-2-439000; Fax : 359-2-875292

16/20 Novembre 1996

Second Asian and Oceanic Congress of Andrology

Chandigarh, Inde

Contact : Dr Natwar R. KALLA - Department of Biophysics

Panjab University, P.O. Box 1204,

Chandigarh 160014, Inde

Tél : 172-541-411 ext : 1354 ; Fax : 172-541-409

12/14 Décembre 1996

XIIlème Congrès de la Société d'Andrologie de Langue Française :

Palais des Congrès, Nancy

Principaux thèmes :

Actualités, mises au point : Maladie de la Peyronie, Biochimie du sperme, Andropause, Hyperthermie testiculaire et infertilité, Adolescence et pathologie de la puberté.

Comportement sexuel mâle, Procréation et paraplégie, Traitements des troubles de l'érection.

Le Testicule :

- Récepteurs des stéroïdes sexuels sur la lignée germinale et leur modulation

- Biologie moléculaire de la méiose, étude de la prolifération goniale

- Fécondation in vitro avec des gamètes immatures

- Réponse du testicule à l'infection

Contact : Pr H. GERARD - Labo. d'Histologie - Embryologie II - Faculté de Médecine 9 avenue de la Forêt de Haye B.P. 184 - 54505 Vandœuvre lès Nancy Cedex Tél : 83-59-28-65 ; Fax : 83-59-28-83 